\title{
CASE-BY-CASE META-ANALYSIS FROM A BAYESIAN PERSPECTIVE
}

\section{Lobna Eid AL-Tyeb}

Faculty of Commerce, Al-Azhar University (Girls' Branch), Egypt

Corresponding author's email: lobnaeid2016@gmail.com 


\title{
116CASE-BY-CASE META-ANALYSIS FROM A BAYESIAN \\ PERSPECTIVE
}

\begin{abstract}
Due to the advancement of science and the tendency to use the smallest of humans in needless clinical trials in many situations, the role of meta-analysis to summarize published study with the same objective, using statistics is becoming more and more fundamental. The integration of the knowledge available makes it easier to understand and allows strong conclusions. For example, increasing the number of clinical trials encourages a rising need to conduct metaanalyses, making complexity possible. The purpose of the research was therefore to suggest a Bayesian meta-analytical methodology. Bayesian method was proposed to carry out a case-by-case metaanalysis. The procedure was based on the mixture of the posterior distributions of the parameter in question from each study that is a percentage of the meta-analysis, thus producing a combined posterior distribution as a meta-analytical measure. In addition, a brief comparison is made between the proposed method and some methods used in meta-analysis. Some examples are presented and discussed.
\end{abstract}

Keyword: Bayesian inference, meta-analysis, combined analysis, DerSimonian and Laird model

\section{Introduction}

The role of meta-analysis is to summarize published studies on a specific problem, using statistics; thus, each day is more fundamental due to the advancement of science and the growth in the number of publications. Systematic reviews and meta-analyses are considered to be important instruments in the evaluation of medical and useful therapies, when there is a desire not to ignore much of the available evidence. 
Haidich A. B. (2010), de ne meta-analysis as an analysis, that is, a statistical analysis that aims to combine results already found in previous analyses of different studies of the same interest. The metaanalysis aims to combine studies carried out under different conditions, with different levels of precision and by groups of researchers from different regions and backgrounds. Thus, broader conclusions are expected than those obtained by the studies that make up the systematization see Ried K. (2006) .

When a meta-analysis is well planned and executed, it is a powerful tool for syntheses; it is an analytical method, in which all studies are integrated and their results grouped in a single conclusion. Thus, when the meta-analysis is compared to other forms of reviewing individual studies, it has the great advantage of being less influenced by the researcher's personal opinion, providing impartial conclusions. In addition, in a meta-analysis, all results from the individual studies examined are reported, and the reader can easily recalculate them and compare them with the conclusions obtained by Gopalakrishnan, S., \& Ganeshkumar, P. (2013).

The meta-analysis based on the literature, because it only works with the results of the studies, can lead us to biased and, often, wrong conclusions. Thus, it is preferable to use meta-analysis on a case-bycase basis, since it works with the raw data of each individual; this type is more informative and allows any type of data analysis, including analyses with data that depend on time, providing more accurate results. On the other hand, there are circumstances in which data from the studies in question cannot be accessed for simple reasons, such as lack of collaboration or loss of data, making it impossible to use this technique see, Ried K.,(2006).

The objective of this paper is to present a Bayesian meta-analytic measure based on the mixture of a posteriori distributions of the parameter of interest for each study belonging to the meta-analysis. 
That is, this proposed meta-analytic measure is a probability distribution of the quantity of interest and not just a simple summary measure of it, leading to a better conclusion of the problem.

This paper contains the methodology for producing the proposed Bayesian meta-analytical measure, presents two examples with metaanalysis data using the proposed methodology. The first example uses proportion as a measure of effect and the second, the difference between averages

\section{Methodology}

Since the posterior distribution for the amount of interest represents, under the Bayesian approach, the most complete inference that can be made about it, the statistical proposal of this work to solve problems in meta-analysis is to mix the posterior distributions of the parameters of interest for each study belonging to the meta-analysis. As the Bayesian operation is often difficult to perform, numerical methods and the approximate Monte Carlo simulation method via Markov Chains are used.

Minjie Xu, Jun Zhu, and Bo Zhang. (2012) introduce the Bayesian paradigm is based on specifying a probabilistic model for the observed data $D$, given a vector of unknown parameters $\theta$, leading to the likelihood function $L(D, \theta)$. It is assumed that $\theta$ is random, with a priori function denoted by $\pi(\theta)$. The inference about $\theta$ is based on the posterior distribution, obtained via Bayes' theorem. The posterior distribution of $\theta$ is given by:

$\pi(\theta \mid \mathrm{D})=\frac{L(D, \theta) \pi(\theta)}{\int_{\Theta} L(D, \theta) \pi(\theta) d \theta}$

where $\Theta$ denotes the parametric space of $\theta$. From (1), $\pi(\theta D)$ is proportional to the multiplication of the likelihood function and a priori, 
$\pi(\theta \mid D) \propto L(D, \theta) \pi(\theta)$

Involving the contribution of the data observed through $\mathrm{L}(\mathrm{D}, \theta)$ and the contribution of a priori information quantified by $\pi(\theta)$. The quantity $m(D)=\int \Theta L(D, \theta) \pi(\theta) d \theta$ is the normalizing constant of $\pi(\theta D)$ and is often called the marginal data distribution or predictive distribution, see Ibrahim, JG; Chen M-H; Sinha, D (2005).

To simplify and introduce the idea of the proposed method, consider an example of a meta-analysis of two studies.

Example 1: Suppose that the variable of interest is normally distributed with mean $\mu$ and variance $\sigma^{2}$. Data from study 1 were generated from a Normal distribution with mean -3 and variance 15 , $\mathrm{X} \sim \operatorname{Normal}(-3 ; 15)$, and from study 2 , from a Normal distribution with mean Example 1: Suppose the variable of interest is normally distributed with mean $\mu 3$ and variance 12 , $\mathrm{Y} \sim \operatorname{Normal}(3 ; 12)$. The sample size of both studies is equal to $30\left(\mathrm{n}_{\mathrm{x}}=\mathrm{n}_{\mathrm{y}}=30\right)$. The mean and sample variance of study 1 are $\bar{x} \sum_{i=1}^{n_{x}} \frac{x_{i}}{n_{x}}=-2.98$ and $s_{x}^{2}=\frac{\sum_{i=1}^{n_{x}}\left(x_{i}-\bar{x}\right)^{2}}{n_{x}-1}=18.04$ and study 2 are, respectively $\bar{y} \sum_{i=1}^{n_{y}} \frac{y_{i}}{n_{y}}=3.35$ and $s_{y}^{2}=\frac{\sum_{i=1}^{n_{y}}\left(y_{i}-\bar{y}\right)^{2}}{n_{y}-1}=$ 8.37 The likelihood function for studies 1 and 2 are, respectively

$$
\begin{aligned}
L_{x}\left(x, \mu, \sigma^{2}\right) & \propto\left(\sigma^{2}\right)^{-\frac{n_{x}}{2}} \exp \left\{-\frac{1}{2 \sigma^{2}}\left[\left(n_{x}-1\right) s_{x}^{2}+n_{x}(\mu-\bar{x})^{2}\right]\right\} \\
L_{y}\left(y, \mu, \sigma^{2}\right) & \propto\left(\sigma^{2}\right)^{-\frac{n_{y}}{2}} \exp \left\{-\frac{1}{2 \sigma^{2}}\left[\left(n_{y}-1\right) s_{y}^{2}+n_{y}(\mu-\bar{y})^{2}\right]\right\}
\end{aligned}
$$

Michael I. Jordan (2010), Considers a priors of Jeffreys ${ }^{1}$ for $\left(\mu, \sigma^{2}\right), \pi$ $\left(\mu, \sigma^{2}\right) \sigma^{2}-1$, and the likelihood function in (3) and (4), the posterior distributions of $\mu$ and $\sigma^{2}$ in both studies follow the Normal-GammaInverse distribution, given by:

$$
\pi\left(\mu, \sigma^{2} \mid x\right)=\frac{\sqrt{n_{x}}}{\sigma \sqrt{2 \pi}} \frac{\left[\frac{\left(n_{x}-1\right) s_{x}^{2}}{2}\right]^{\frac{n_{x}}{2}}}{\Gamma\left(n_{x} / 2\right)}\left(\frac{1}{\sigma^{2}}\right)^{\frac{n_{x}+1}{2}+1}
$$


$\exp \left\{-\frac{\left(n_{x}-1\right) s_{x}^{2}+n_{x}(\mu-\bar{x})^{2}}{2 \sigma^{2}}\right\}$

$\pi\left(\mu, \sigma^{2} \mid y\right)=\frac{\sqrt{n_{y}}}{\sigma \sqrt{2 \pi}} \frac{\left[\frac{\left(n_{y}-1\right) s_{y}^{2}}{2}\right]^{\frac{n_{x}}{2}}}{\Gamma\left(n_{y} / 2\right)}\left(\frac{1}{\sigma^{2}}\right)^{\frac{n_{y}+1}{2}+1}$

$\exp \left\{-\frac{\left(n_{y}-1\right) s_{y}^{2}+n_{y}(\mu-\bar{y})^{2}}{2 \sigma^{2}}\right\}$

That is $\left(\mu, \sigma^{2} \mid x\right) \sim \operatorname{NIG}\left(\bar{x}, n_{x}, \frac{\left(n_{x}-1\right)}{2} \frac{\left(n_{x}-1\right) s_{x}^{2}}{2}\right)$ and $\left(\mu, \sigma^{2} \mid y\right) \sim$ NIG $\left(\bar{y}, n_{y}, \frac{\left(n_{y}-1\right)}{2} \frac{\left(n_{y}-1\right) s_{y}^{2}}{2}\right)$. The values expected a posteriori and the a posteriori variance-covariance matrix of $\mu$ and $\sigma 2$ for (5) are, respectively,

$$
\begin{aligned}
& E\left[\mu, \sigma^{2} \mid x\right]=\left(\frac{\left(n_{x}-1\right) s_{x}^{2}}{n_{x}-3}\right), n_{x}>3 \\
& \operatorname{Cov}\left[\mu, \sigma^{2} \mid x\right]=\left[\begin{array}{c}
\frac{s_{x}^{2}\left(n_{x}-1\right)}{n_{x}\left(n_{x}-3\right)} \\
0
\end{array} \frac{2 s_{x}^{2}\left(n_{x}-1\right)}{\left(n_{x}-5\right)\left(n_{x}-3\right)^{2}}\right] \quad n_{x}>5
\end{aligned}
$$

The values expected a posteriori and the variance-covariance matrix of $\mu$ and $\sigma^{2}$ for model (6) are analogous to (7) and (8). It is worth mentioning that the joint posterior distribution of $\mu$ and $\sigma 2$ depends on the data only by means of sufficient statistics $\mathrm{n}_{\mathrm{x}}, \bar{x}$, $\mathrm{s}_{2}$ and ny, $\bar{y}$, $\mathrm{s}_{2}$, that is, the evidence provided by the sufficient statistics is identical to that provided by the data .In this case, performing a meta-analysis based on the literature is equivalent to performing a meta-analysis on a case-by-case basis, without loss of quality. Using (5) and (6), it is suggested to mix the posterior distributions of $\left(\mu, \sigma^{2}\right)$ of each study. This is,

$$
\pi_{M}\left(\mu, \sigma^{2} \mid x, y\right)=\frac{n_{x} \pi\left(\mu, \sigma^{2} \mid x\right)+n_{y} \pi\left(\mu, \sigma^{2} \mid y\right)}{n_{x}+n_{y}}
$$


In Figure.1 (a), are the level curves of the a posteriori joint distribution of $\mu, \sigma^{2}$ for each study, in order to observe threedimensional graphs in only two dimensions. It is observed that the difference between the a posteriori averages seems large. In Figure.1 (b), the level curves of the distribution for the posteriori meta-analytic distribution of $\mu, \sigma^{2}$, which is given by mixing the distributions of the a posteriori meta-analytic distribution given in (9). In other words, the proposed meta-analytic measure is a distribution of $\Sigma \mu, \sigma^{2}$ and not just a simple summary measure. In this case, the measure required a better investigation of the problem. It is worth mentioning that the regions with the highest density in graphics are those most likely to meet the "true" parameters. The estimation by region HPD (Highest Posterior Density) with 95\% of credibility is in Figure.1 (d). HPD is less than or equal to $95 \%$. Region / range that contains the true value of the parameter of interest, with greater probability at 0.21 with a $95 \%$ confidence interval equal to $[5.99 ; 6.41]$. The results of this meta-analysis, if the meta-analytic al measure were calculated using the DerSimonian and Laird method see Jackson, D., Law, M.et all (2016), this would be the same based on the literature are summarized in the Forest Plot graph, Figure.2, Meta-analytic is given in (10). However, one wonders: does this make sense? Making a metaphor, A brief review of the method, for better understanding, is presented later and the estimator

result found is the same as saying that, having your head in the freezer and with your feet in the oven, on average, the temperature is nice. 

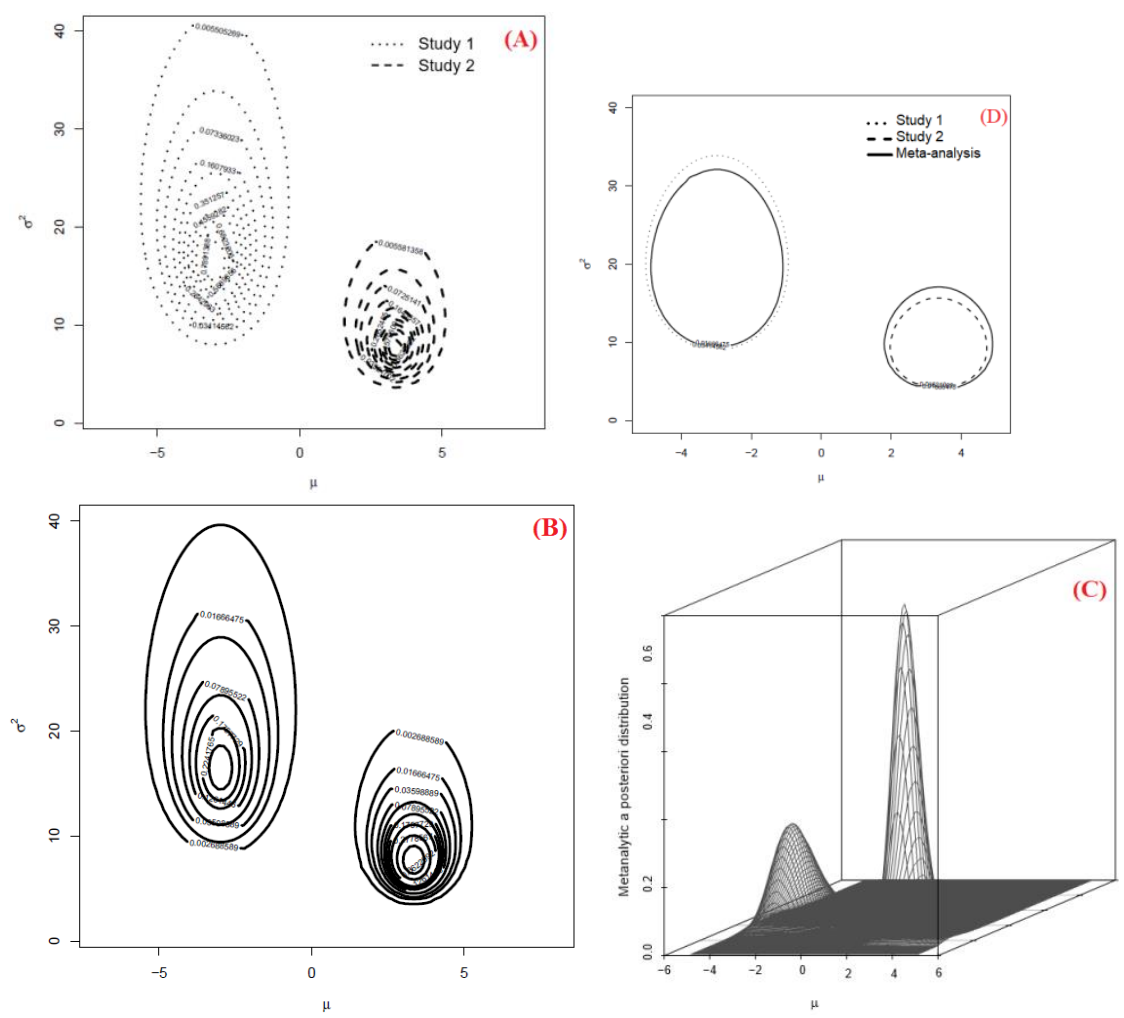

Figure.1: (a) Level curves of the posterior joint distribution of $\left(\mu, \sigma^{2}\right)$ in each study. (b) Level curves of the a posteriori meta-analytic al distribution of $\left(\mu, \sigma^{2}\right)$. (c) Three-dimensional graph of the a posteriori meta-analytic al distribution of $\left(\mu, \sigma^{2}\right)$. (d) $95 \%$ credibility HPD region for $\left(\mu, \sigma^{2}\right)$.

\begin{tabular}{|c|c|c|c|}
\hline Study & & & Median (CI 95\%) \\
\hline Study 1 & $\diamond$ & & $-2.98[-4.50,-1.46]$ \\
\hline Study 2 & & $\diamond$ & $3.35[2.31,4.39]$ \\
\hline RE model & 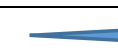 & & $0.21[-5.99,6.41]$ \\
\hline-6.00 & -2.00 & 2.00 & 6.00 \\
\hline
\end{tabular}


Figure.2: Forest Plot for the data in Example 1.

In order to try to approximate the methods for possible comparisons, a posteriori meta-analytic distribution is found only in terms of $\mu$. The marginal posterior distributions of $\mu$, in the studies 1 and 2 are $\mu \mid \mathrm{x} \sim$ $\mathrm{t}_{\mathrm{n}-1} \bar{x}, s_{x}^{2} / n x$ and $\mu \mid \mathrm{y} \sim \mathrm{t}_{\mathrm{n}-1} \bar{y}, s_{x}^{2} / n y$. To find the meta-analytic al measure of $\mu$. It is important to note that the $95 \%$ credibility distribution given by the disjunction intervals $[4.55 ; 1.41]$ and $[2.17$; 4.53]. Also the marginal meta-analytic posteriori of $\mu$ is also a bimodal distribution with HPD interval. It can be observed that the meta-analytic value found by the DerSimonian and Laird method is outside the HPD ranges. In this case, the meta-analytic al distribution is bimodal and finding the a posteriori mean of $\mu$ does not provide much information. The proposed measure suggests the existence of two distinct groups, which leads us to think better about the problem and the possible combination of these studies see Veroniki, A. A., Jackson, D., et all(2016).

Suppose, furthermore, that only the result of the usual meta-analysis, the traditional Forest Plot diamond, and the result of the proposed meta-analysis, the a posteriori meta-analytical distribution, are available. If only these results are available, it is clear that the result obtained by the proposed method allows for more comprehensive conclusions and is more informative than usual. Therefore, using a probability distribution as a meta-analytical measure is more plausible, since one can observe the behaviour of the parameter in question. It is worth mentioning that, in this example, there is also the advantage that the results obtained by the proposed method, considering the sufficiency statistics, are the same obtained by the case-by-case meta-analysis. An important observation is that this measure can also find similarities between studies and differences between the groups suggested by this distribution, which can be caused by some factor not investigated, showing an advantage when 
using this measure. It is important to make it clear that the objective is not to compare methods, as they are not comparable. However, the aim is to highlight possible flaws in a methodology, which is widely used in meta-analysis and to draw attention to how the same problem can be solved. The DerSimonian and Laird method is nothing more than a weighted average of the measures of effect of the studies. In the method, there is no assumption of homogeneity between studies. The meta-analytic al measure is given by:

$\hat{\theta}_{M D L}=\frac{\sum_{j=1}^{J} w_{j}^{*} \hat{\theta}_{j}}{\sum_{j=1}^{J} w_{j}^{*}}$

Where, $w_{j}^{*}=\frac{1}{\tau^{2}+w_{j}^{-1}} w_{j}^{*}=\frac{1}{\sigma_{j}^{2}}, \tau^{2}=\frac{Q-j-1}{\sum_{j=1}^{J} w_{j}-\frac{\sum_{j=1}^{j} w_{j}^{2}}{\sum_{j=1}^{J} w_{j}}} Q=\sum_{j=1}^{J} w_{j}\left(\hat{\theta}_{j}-\widehat{\theta}_{M}\right)^{2}, \widehat{\theta}_{M}=$ $\frac{\sum w_{j} \widehat{\theta}_{j}}{\sum w_{j}}, \hat{\theta}_{j}$ is the measure of effect of the $j^{\text {th }}$ study and $\tau$ is the estimated variability between studies, $\mathrm{j}=1.2, \hat{\tau}^{2}$ can be less than zero and, if this happens, $\hat{\tau}^{2}=0$ and the metanalytic measure is the same as the calculated by the Mantel-Haenszel method. The 95\% confidence interval for $\hat{\theta}_{M D L}$ is given by Ahn, E., \& Kang, H. (2018), $\hat{\theta}_{M D L}=$ $\pm 2 / \sqrt{\sum_{j=1}^{J} w^{*}}$.

Another important aspect in the meta-analysis to be highlighted is that it should not be confused with combined analysis. The combined analysis of data disregards the study factor, uniting all individuals in a single sample and considering a meta-analysis, as if it were a large study, is to lose all the existing variability between them. The comparison between the combined analysis and the meta-analysis, for the data in this example, can be seen in Figure 2.3. Ignoring, then, the fact that the data come from different studies, a single sample of size 60 is assumed. From this single sample, the mean is equal to 0.18 and the standard deviation of 4.81. $\left(\mu, \sigma^{2}\right)$ as in (5) and the posterior posterior distribution of $\mu$, represented in Figure.3 (a) and Figure.3 
(b). The a posteriori mean of $\mu$ is 0.18 with an HPD interval of $95 \%$ of credibility in the Bayesian approach described above, the joint posterior distribution of equal to $[1.06 ; 1.42]$. Note that the combined analysis differs considerably from the proposed meta-analysis, which does not occur with the meta-analysis via the DerSimonian and Laird method.
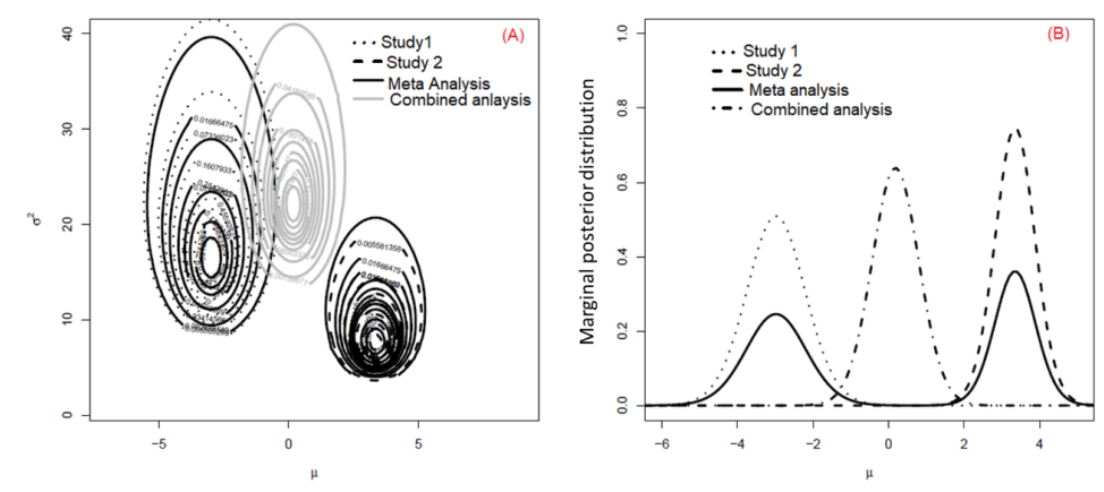

Figure.3: (a) Level curves of the joint posterior distribution of $\mu$ and $\sigma^{2}$. (b) Marginal posterior distribution of $\mu$.

2.1 Generalization of the proposed method

Interest $\theta$ For the $j^{\text {th }}$ study $(j=1, \ldots J)$, suppose that $x_{j}=\left(x_{1}, j, x_{2}, j\right.$, $\left.\ldots, x_{n}, j\right)^{T}$ denotes a vector of random variables of size $n_{j}$, with probability density function $f\left(x_{j}, \theta\right)$. Consider a meta-analysis of $J$ independent studies that investigate a certain characteristic of in the probability space $(X, F, P)$. Considering $x_{j}=\left(x_{1}, j, x_{2}, j, \ldots, x_{n j}, j\right)$ an observed random vector of $x_{j}$, the likelihood function for the $j^{\text {th }}$ study is given $L_{j}\left(x_{j}, \theta\right)=\prod_{i=1}^{n_{j}} f\left(x_{i j}, \theta\right)$. Admitting the Bayesian methodology, it is observed, respectively, the a-funi $i=1$ a priori and the a posteriori distribution of $\theta, \pi(\theta)$ and $\pi_{j}\left(\theta \mid x_{j}\right) L_{j}\left(x_{j}, \theta\right) \pi(\theta)$. In general, the a priori function is the same for all studies, as it does not depend on where the experiment was conducted. Then, the proposed meta-analytic measure is the mixture of $J$ posterior distributions of $\theta$, producing a single distribution for $\theta$, which is called the posterior 
meta-analytic distribution of $\theta$. Density mixing is a technique used in some cases to derive conclusions about $\theta$ see Haynes, M., Mengersen, K. (2005) . Therefore, the a posteriori meta-analytic al distribution of $\theta$ is given by Eq. 11

$\pi_{M}(\theta \mid x)=\sum_{j=1}^{J} w_{j} \pi_{j}\left(\theta \mid x_{j}\right) \propto\left[\sum_{j=1}^{J} w_{j} L_{j}\left(x_{j}, \theta\right)\right] \pi(\pi)$

where $w_{j}$ is the weight of the $j^{t h}$ study. These weights are fixed and can represent the importance of the studies, the sample size, etc. Initially, consider $w_{j}=\frac{n_{j}}{\sum_{j=1}^{J} n_{j}}$. The a posteriori mean meta-analytic and the a posteriori meta-analytic variance for $\theta$ are, respectively,

$E_{M}(\theta \mid x)=\sum_{j=1}^{J} w_{j} E_{j}(\theta \mid x)$

$\operatorname{Var}_{M}(\theta \mid x)=\sum_{j=1}^{J} w_{j}^{2} \operatorname{Var}_{j}\left(\theta \mid x_{j}\right)$

The proposed meta-analytic measure is a general measure, that is, any a priori function and any likelihood function can be used in (11). What makes it possible to use it, from the most trivial meta-analysis problem to the most complex ones? It is also worth mentioning that, when sufficient statistics for $\theta$ is available, performing a meta-analysis based on the literature will be equivalent to performing a metaanalysis on a case-by-case basis, without loss of information.

It is important to make it clear, in order to have a quality metaanalysis, in addition to an appropriate statistical technique, it is important that the studies to be combined have been carefully evaluated, so that there is confidence in their association. Therefore, it is necessary that study selection criteria are established see Russo M. W. (2007). 
For Egger and Smith (2009), meta-analysis expresses cautious planning. The prior definition of qualifying criteria for studies is included in a detailed search, in order to guarantee a high quality of the meta-analysis. There are different statistical methods for combining the data; however, there is no single "correct method". Although the studies belonging to the meta-analysis involve the same subject, there are differences between them, which is called heterogeneity between the studies, this is one of the usual problems in meta-analysis and one solution is to incorporate it into the statistical analysis. Statistical models that incorporate the variability component between studies are widely discussed, both in classical and Bayesian theory. Berry (1989) considers the formulation of a Bayesian hierarchical model to describe and explain the heterogeneity that exists between individual studies, and classical theory involves combining the magnitude of the effects. Another model that has been used to analyse the heterogeneity existing between the studies, is the Nita mixture model suggested by Schlattmann (2009).

Bayesian methods are more flexible than classic ones. The data affect the Bayesian inference only by the likelihood function, that is, the stopping rule and other characteristics of the study design are irrelevant. Such methods also offer a convenient structure for incorporating the information available a priori and are applicable to problems that combine studies with small sample sizes, when Bayesian estimators are more accurate than those obtained through maximum likelihood. An important aspect to be highlighted is that the method proposed in this work does not discuss the question of heterogeneity or homogeneity between the studies, since the variability between the studies included in the meta-analysis is always taken into account see Etzioni, Ruth \& Kadane, Joseph. (1995). 


\section{The results and Conclusion are summarized in the following aspects:}

A Bayesian meta-analytic al measure was proposed as an alternative to the methods used. This measure is a mixture of a posteriori distributions of the parameter of interest, $\theta$, for each study. The proposed method produces a posteriori probability distribution of $\theta$ as a meta-analytic al measure. Through this distribution, the hope and a posteriori variance of $\theta$ can be calculated, in addition to other measures. It was observed that the proposed methodology is a general measure, making it possible to be used, from the most trivial metaanalysis problem to the most complex ones. In addition, when sufficient statistics are available, performing a meta-analysis based on the literature is equivalent to performing a meta-analysis on a case-bycase basis, without loss of information. This results in a quality gain for meta-analysis based on the literature, since case-by-case metaanalysis is considered the gold standard.

The proposed methodology was used to solve meta-analysis problems with survival data. It was observed that, for the Exponential and Log-normal models, performing case-by-case meta-analysis with survival data is equivalent to doing a meta-analysis based on the literature, which seemed difficult in analyzes with these types of data. Weibull model was used to model life span. The survival curves and their credibility bands were estimated via Metropolis-Hastings, as well as the meta-analytic survival curve.

The method usually used to perform a meta-analysis based on the literature, is based on the DerSimonian and Laird model. This method incorporates the variability that exists between the studies in the weighting of the measures of effect of each study to then produce a meta-analytic al measure. Such a method tends to give greater weight to small studies, since these studies may reflect a publication bias, the use of this model can emphasize poor evidence at the 
expense of good evidence. Furthermore, it was observed that the resulting meta-analytic al measure is poor, can be biased and does not provide much information about the parameter in question. The Nita mixture model used in meta-analysis presented difficulties in interpreting the results and, in addition, it did not take into account the variability that existed between the studies. The hierarchical model also left something to be desired in the use of all available information, ignoring important points of the problem presented. However, such problems did not occur with the proposed Bayesian meta-analysis.

The proposed meta-analytic al measure is a mixture of a posteriori distributions of the parameter of interest, $\theta$, from each study. It is important to note that this method produces a probability distribution as a meta-analytic al measure, called the $\theta$ meta-analytic posteriori distribution. Through this distribution, the hope and a posteriori variance of $\theta$ can be calculated, in addition to other measures. Therefore, the proposed methodology is much more informative, intuitive and precise than the existing ones. Several works may be developed based on the ideas and results of this research work. Some of them are cited:

- Computational improvement to carry out the proposed metaanalysis;

- Applications in other types of statistical models; 


\section{References}

Ahn, E., \& Kang, H. (2018). Introduction to systematic review and meta-analysis. Korean journal of anesthesiology,71(2), 103112. https://doi.org/10.4097/kjae.2018.71.2.103

Berry, DA (1989). A Bayesian Approach to Multicenter Trials and Meta-analysis. National Science Foundation, Washington, D.C

Egger, M; Smith, GD; Altman, DG (2009). Systematic Reviews in health care. Meta-analysis in context. BMJ books.

Etzioni, Ruth \& Kadane, Joseph. (1995). Bayesian Statistical Methods in Public Health and Medicine. Annual review of public health. 16. 23-41. 10.1146/annurev.pu.16.050195.000323.

Glass, G. V. (1976). Primary, secondary, and meta-analysis of research. Educational Researcher,5(10), 3-8

Gopalakrishnan, S., \& Ganeshkumar, P. (2013). Systematic Reviews and Meta-analysis: Understanding the Best Evidence in Primary Healthcare. Journal of family medicine and primary care, 2(1), 9-14. https://doi.org/10.4103/2249-4863.109934

Haidich A. B. (2010). Meta-analysis in medical research. Hippokratia, 14(Suppl 1), 29-37.

Haynes, M., Mengersen, K. Bayesian estimation of $g$-and$k$ distributions using MCMC. Computational Statistics 20, 7-30 (2005). https://doi.org/10.1007/BF02736120

Ibrahim, JG; Chen M-H; Sinha, D (2005). Bayesian Survival Analysis. Springer Series in Statistics, Second Ed.

Jackson, D., Law, M., Barrett, J. K., Turner, R., Higgins, J. P., Salanti, G., \& White, I. R. (2016). Extending DerSimonian and Laird's 
methodology to perform network meta-analyses with random inconsistency effects. Statistics in medicine, 35(6), 819-839. https://doi.org/10.1002/sim.6752

Michael I. Jordan, Lecture 7: Jeffreys Priors and Reference Priors, Lecture Date: $\quad$ February $17, \quad 2010$ https://people.eecs.berkeley.edu/ jordan/courses/260spring10/lectures/lecture 7.pdf

Minjie $\mathrm{Xu}$, Jun $\mathrm{Zhu}$, and Bo Zhang. Bayesian nonparametric maxmargin matrix factorization for collaborative prediction. In Advances in Neural Information Processing Systems, pages 6472,2012

Ried K. Interpreting and understanding meta-analysis graphs--a practical guide. Aust Fam Physician. 2006;35:635-8

Russo M. W. (2007). How to Review a Metaanalysis. Gastroenterology \& hepatology, 3(8), 637-642.

Schlattmann, P (2009). Medical Applications of nite mixture models.

Statistics for Biology and Health, Springer

Veroniki, A. A., Jackson, D., Viechtbauer, W., Bender, R., Bowden, J., Knapp, G., Kuss, O., Higgins, J. P., Langan, D., \& Salanti, G. (2016). Methods to estimate the between-study variance and its uncertainty in meta-analysis. Research synthesis methods, 7(1), 55-79. https://doi.org/10.1002/jrsm.1164 